\title{
Museum Navigation based on NFC Localization Approach and Automatic Guidance System
}

\author{
Dawei Cai \\ Iwate Prefectural University \\ Iwate, Japan 020-0193
}

\begin{abstract}
This paper presents an automatic museum guide system that provides both interactive guidance for exhibition and a NFC based location navigation. It is well known that NFC is a kind of short distance communication for portable terminals such smart phones and tablet devices. The communication area of NFC is limited in a narrow area; therefore, NFC may be used to locate the position of the area. To realize the automatic guidance and navigation, we present a new data architecture to contain the location information, and an evaluation function to calculate the optical exhibition candidates for the visitors. The feature of the function is considering the popularity of the exhibition and movement distance between the exhibition spots. To implement the service, we developed an application to support editing the map, and create the map data for the portable terminals. It is confirmed that the system provide a good performance based on the experiment in museum.
\end{abstract}

\section{General Terms:}

System Development

\section{Keywords:}

NFC, tag, GUI, optimization, museum guidance, navigation system

\section{INTRODUCTION}

Museum visitors' guides systems with ICT have recently been attracting a growing interest and firing more research efforts and business activities. There are several museum guidance systems with general smart phone or digital device such as iPad, iPod touch, or portable game player such as Nintendo 3D in [1],[2],[3]. Most of them can provide a multimedia guidance, but several problems may block their wide usages. For example, the huge multimedia contents will be downloaded to the phone through network, but it will be cost a lot for foreign tourists. The guidance cannot be provided to the visitor who does not have the phone or the device. Another problem is traffic trouble if many visitors access the same contents at the same time. Therefore, a cheap and specialized device for the museum is necessary for most cases. As reported in [4] and [5], we developed an infarred communication to transmit the content in wireless mode with a high performance. A cheap and high performance portable guidance device and its application system to provide good guidance service for both visitors and museum management. The new device is very light and easy to use, can run for more than 8 hours with a full recharge.

For some museum with large or complex exhibition areas, it may be important for visitors to know their current position or the places they have visited or will visit. If the visitor can confirm the above position information or visiting history information, they may arrange their visiting plan with a better efficiency and get more enjoyment or satisfactory for the exhibition. At the base of the previous platform, we developed a new navigation function that can show the current position and visiting history information.

This paper presents a new guidance system that not only provides multimedia guidance for the visitors, but also location navigation based on NFC information. In most position system, GPS or WiFi signal are used to locate the device position, but we use NFC signal to locate the device position instead. The merit of our approach is that the NFC may provide position information at a low cost and easier realization and better accuracy possible, also a lower battery consumption performance than others methods. To support the location information management, we developed a map management application that can edit the NFC tag position and exhibition position easily, and create the map data for the portable terminals automatically. There is a device management function developed to provide powerful and efficient device management services such as updating the contents in the device, obtaining the users' operation historic information, monitoring the charging status. With these functions, the work for the facility staffs can be reduced greatly.

\section{INTERACTIVE MUSEUM GUIDE SYSTEM}

\subsection{System Overview}

Fig 1 shows the main configuration of the new guide system. As shown in Fig 1 there are two sub systems. One is for management department and one is for visitors in exhibition area. The first sub system is composed of integrated device management station, content editor, map creator and stamp rally analyzer. The integrated device management station provides battery charging, contents installation and modification, and action mode setting for device. It will read the usage history from the devices and give a statistics report about the action of the visitors. The content editor with an easy use interface, supports to edit multimedia contents and output the data for the device automatically. The map creator is used to register the exhibition spot and tag spot information to the map 


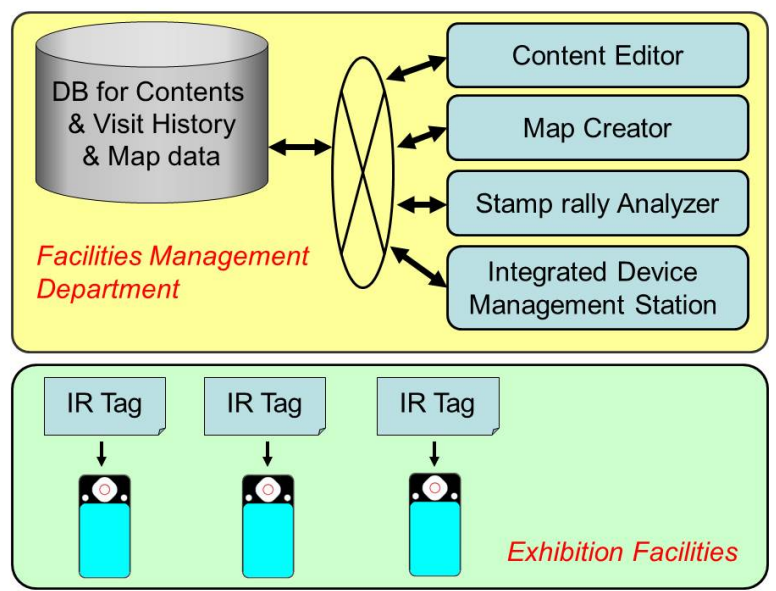

Fig. 1. The basic architecture of the guide system

according to a special map data structure, which will be introduced later in detail. With the map data, the device can show visitors the current location and history of the visiting.

In the first sub system, we developed an Integrated Device Management Station that can send contents and map data and setting data to the portable terminal, and collect visiting history data from the terminals with a special wireless communication that use both infrared communication and a radio communication. The infrared communication uses a new protocol we developed, and can send data to many devices at the same time. With the new communication protocol, the transmitting time can be reduced greatly.

Easy to use contents editor software is also developed to support creating contents for ones who do not have much knowledge about the computer. The user can create content with only simple mouse and keyboard operation. Also, the software will output the content file for the device automatically.

To realize the navigation service, a map data for exhibition area including the position information of exhibits and the tags is necessary. A map making software is designed and developed. For interactive guidance service, a stamp-rally function is provided and a stamp-rally analyzer can read the visiting history wirelessly and gives the result based on the visiting history reading for the device.

In second sub system, we developed a portable terminal, which equipped with NFC ability to get ID information from NFC tags, such as IR (infrared ) tag, RFID tag or PAN (personal area network) tag. The device use wireless communication to get contents data from the Integrated Device Management Station, and send the usage history to the station. The portable terminal will play the guidance contents while the visitor close to an exhibition spot, where the device will get a NFC Signal from NFC tag, such as RFID tag, IR tag or PAN tag.

\subsection{Portable Terminal for Guidance}

A new portable terminal is developed for providing wireless communication ability and contents playing ability. As the wireless communication, there are a hybrid communication that uses infrared communication and radio communication, a RFID reader,

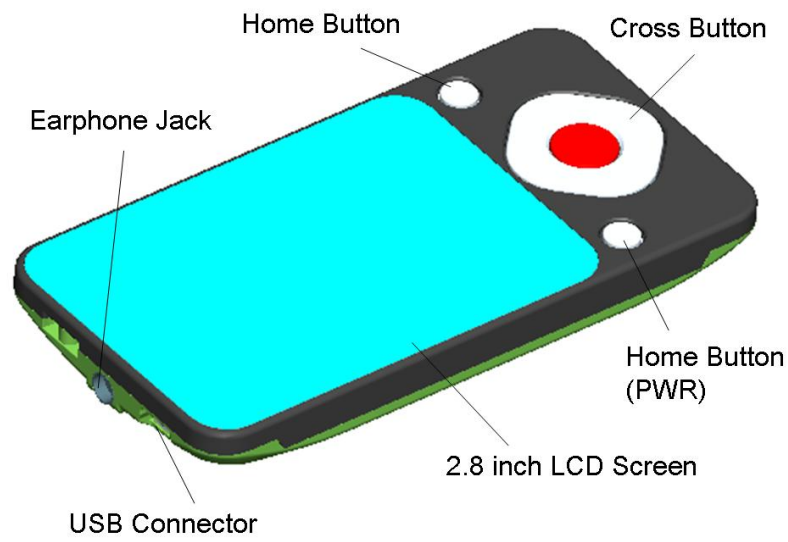

Fig. 2. New portable guide device

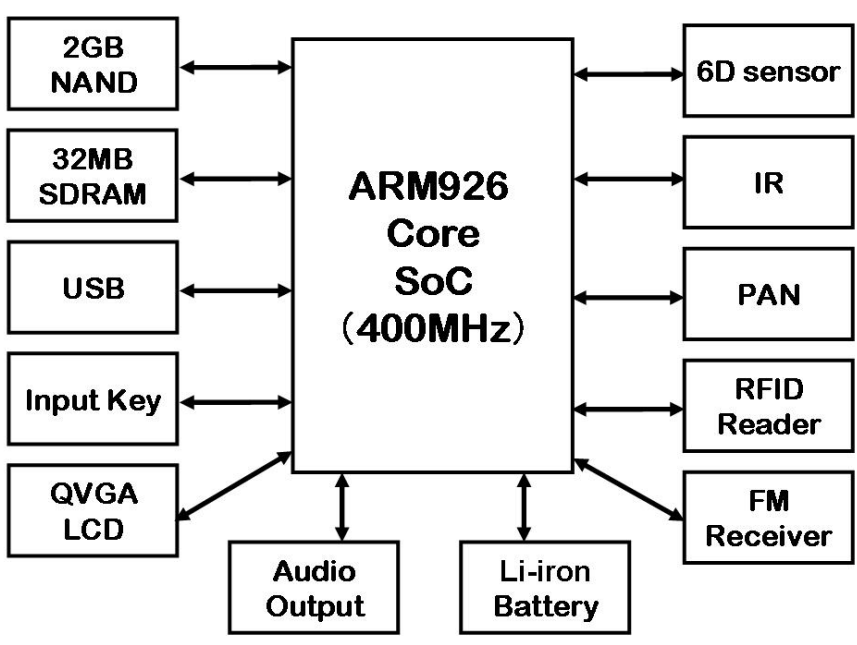

Fig. 3. The Architecture of portable Terminal

IR tag, PAN and FM receiver. The device can play multimedia contents, such as multi-language text, image, audio, and video. There are a cross-key and two additional keys for switching the contents playing or inputting the answers of the quits. In this device we board a 6 axis acceleration and terrestrial magnetism sensor IC to obtain the information about the device orientation or move distance. With the NFC, the portable device can start playing the related content automatically without any operation by users; therefore the device can be used by a wide range visitor from children to elder persons. Fig 2 shows the main parts of the portable device.

The new portable terminal is developed with an ARM926 core SoC and a RTOS platform called ThreadX. Fig 3 shows the main structure of the portable device. As shown in Fig 3 , the portable 


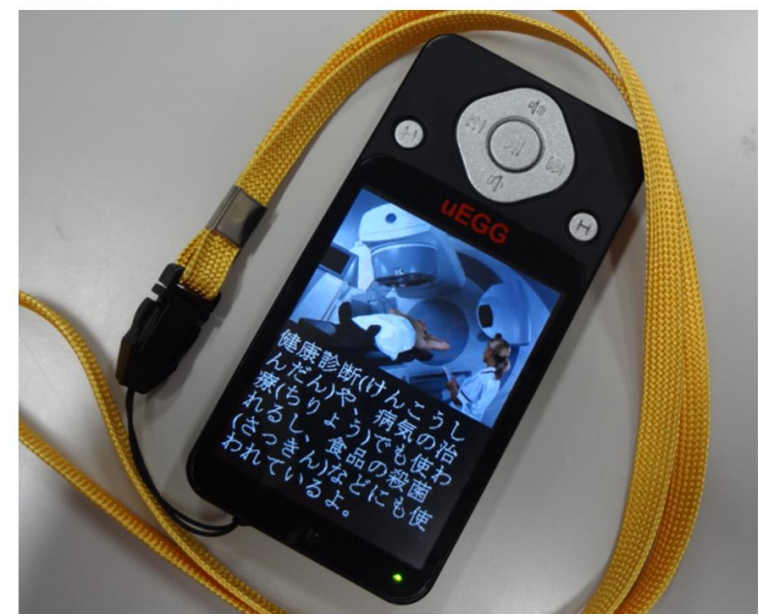

Fig. 4. The portable terminal

Table 1. The specification of the new portable Device

\begin{tabular}{|l|l|}
\hline Communication & $\begin{array}{l}\text { Instant infrared } \\
\text { communication (20Mbps), } \\
\text { Infrared Tag Communication(115Kbps), } \\
\text { RFID Reader (ISO15693) } \\
\text { PAN (2Mbps,2.4GHz) } \\
\\
\text { FM receiver, USB 2.0(HS) }\end{array}$ \\
\hline MEMS sensor & $\begin{array}{l}6 \text { axis acceleration and } \\
\text { terrestrial magnetism sensor }\end{array}$ \\
\hline Flash Memory & $1,2,4,8,16 \mathrm{~GB}$ \\
\hline Support mdeia & MP3, WMA, WMV, MPEG4, H264 \\
\hline Text display & Japanese,English, Chinese, others \\
\hline Displayer & 2.8 inch QVGA LCD, 260K colors \\
\hline Battery duration & 20 hours for audio playing, \\
& 5 hours for video playing, \\
& 40 hours for standby \\
\hline size & $51(\mathrm{~W}) \times 102(\mathrm{~L}) \times 11.3(\mathrm{H}) \mathrm{mm}$ \\
\hline weight & about $70 \mathrm{~g}$ \\
\hline
\end{tabular}

device is composed with a ARM926 core SoC, STMP3760 from FreeScale, 6 degrees sensor IC BCM050 from BOSCH, PAN communication IC NRF24L01 from NORDIC, a flash memory, a MDDR, a 2.8 inch LCD with $0.26 \mathrm{M}$ color and audio output with both earphone and a mini speaker. A 650mAh Li-iron battery is used to provide the power to guarantee the device can work more than 8 hours without charging. Compared with other wireless communication such as WLAN, Bluetooth, the infrared communication and PAN radio communication can reduce the power consumption greatly, therefore, the new portable terminal can provide a much longer operation time. For a guide system in exhibition facilities, it is important that the portable device can provide guidance service without recharging during the whole opening period.

The basic specification of the new portable device is shown as table 1. The new device can provide not only the good performance for showing multimedia contents, but also multi-communication abilities, therefore the new device is expected to have a wide application in various fields.

\subsection{Integrated Device Management Station}

If an exhibition facility uses several tens or hundreds device to provide the guidance service, it may be great heavy work to update the guidance contents with conventional method, which is copying content with USB cable one by one. To provide guidance service to the visitor, the device must be charged and install the necessary contents and action mode data for different usages, such as language selection. As shown in Fig 5 there is a set of charge slots and a communication controller that is connected to $\mathrm{PC}$ with a USB cable. In the station, a hybrid communication, which use both infrared data broadcasting ( $\mathrm{IrDB}$ ) and radio communication called as PAN (personal area network), is presented and implemented in the station. The feature of the communication is that the content will be simultaneously send to the devices, therefore the time for transmitting will not change greatly when the numbers of the device changed greatly. The table 2 shows the main specification of the hybrid communication. Fig 6 shows the hardware and application main window of the Integrated device management station.

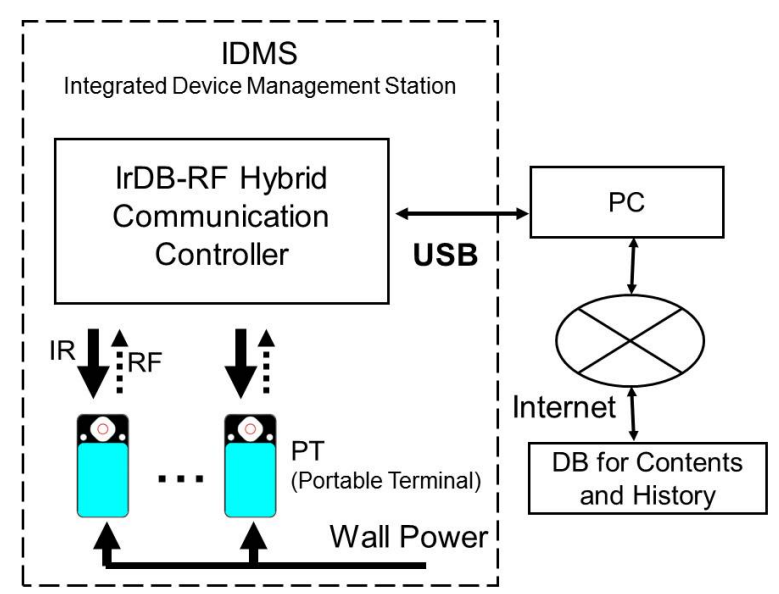

Fig. 5. Architecture of integrated device management station

Table 2. The specification of the hybrid communication

\begin{tabular}{|l|l|l|}
\hline Specification Item & IrDB & PAN \\
\hline Link Direction & Downlink & Uplink \\
\hline Media & Infrared, 680nm & $2.4 \mathrm{GHz}$ radio \\
\hline Rate & $16 \mathrm{Mbps}$ & $2 \mathrm{Mbps}$ \\
\hline Packet Length & $2048 \mathrm{Byte}$ & $32 \mathrm{Byte}$ \\
\hline Communication Distance & $\leq 3 \mathrm{~m}$ & $\leq 10 \mathrm{~m}$ \\
\hline Output Power & $10 \mathrm{~mW}$ & $1 \mathrm{~mW}$ \\
\hline
\end{tabular}




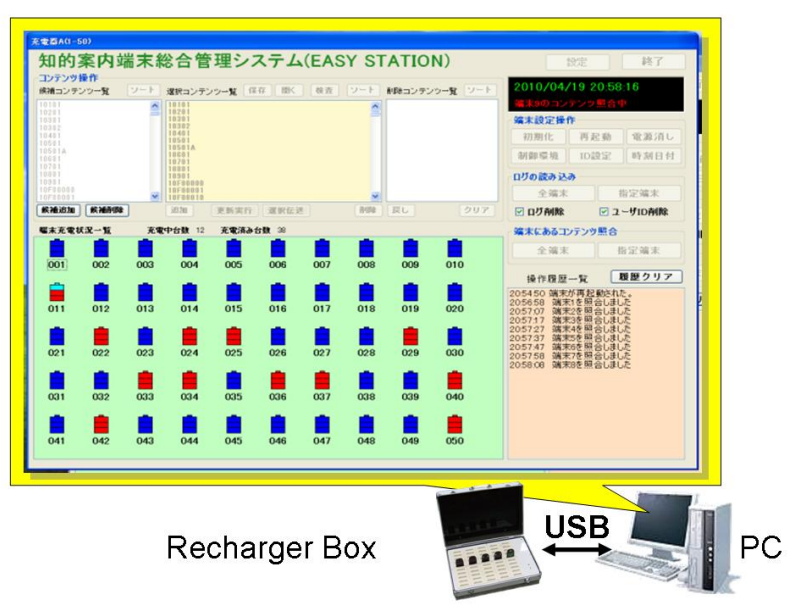

Fig. 6. Integration management station

\subsection{Content Editor}

It may be a money or time consuming to create or update the guidance contents for most exhibition facilities. Here we developed a content editor to make it easier. For creating contents, you only consider the scenario and prepare the material for the contents; you can create or update your contents with only mouse operation or a little keyboard typing. The software will transform the original project date to a smaller contents data for the device automatically. You can modify the content using the project data, or create a new one with the previous data. Fig 7 shows main GUI of the content editor software. The GUI is divided in three area, scene list area, scene editing area, material area. In the scene list area, a sequence scene icon is shown in display order. In scene editing area, the current scene is shown. You can modify the current scene by appending or replacing the image or audio or video data which are listed in material area. After you finished the editing work, we can click the [making] button to create content data for the device. The transforming will be done automatically. If there is any error for making, the program will show the error position for your correction. To make a scene in the contents, first you select a template from the template list, and then edit the template by inserting materials such as images, text, audio and video. The action mode such as scene switching condition or constraint condition for operation can be modified in this mode. A preview function can be used to confirm the playing performance. Therefore, complete contents can be created with this soft. For the screen of the device is only a QVGA type, we do not need a high resolution image for the device. The soft will adjust the image resolution to optimization size to create a final data for the device. By this process, the final data for the device will be reduced, it will reduce the transmission time and memory greatly.

\subsection{Stamp rally Service}

To realize interactive guidance, we present an electronic stamp rally service and developed an evaluation system. Fig 9 shows the GUI of the evaluation software. In the GUI, the left area is used for lending management which can record the lending timing and returning timing automatically, and give a device list for lending device. The right area is used to show the evaluation result of the quiz answers. Also a participation certificate can be printed at once.

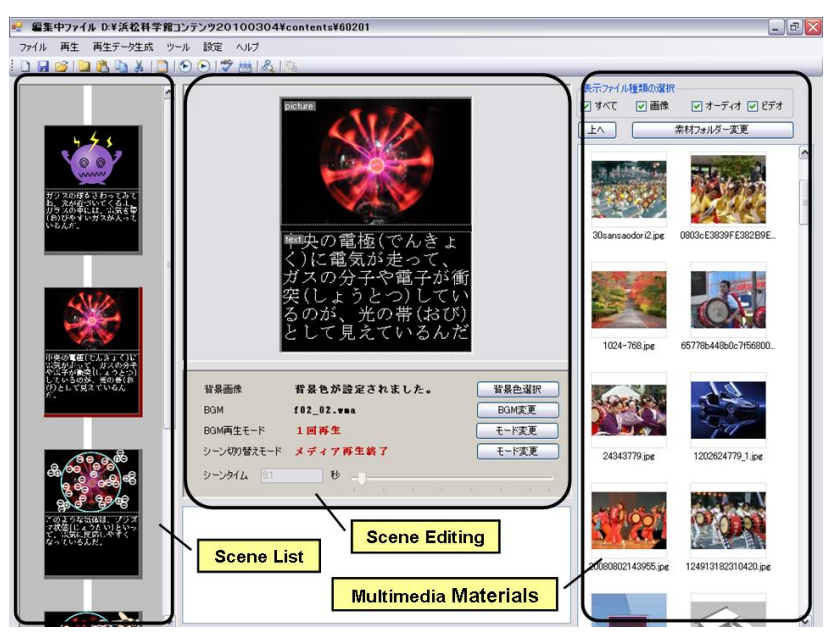

Fig. 7. GUI of the content editor

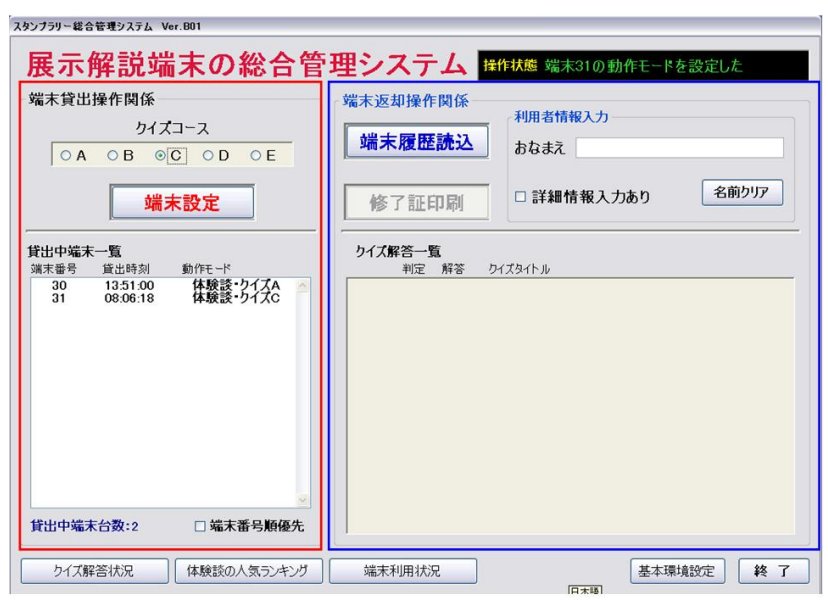

Fig. 8. GUI of the stamp rally evaluator

This soft also provide a statistics function about the usage of the contents and device. The statistics function of the contents will calculate the usage status of contents, and give the lending status, such as lending time, lending times, etc.

As stamp rally, there are five courses with 10 quizzes each. The device will be set to one the five courses. The device shows 10 quiz icons on its desktop, and the icon will change if the quiz is answered. When all the icons changed, it means that all quizzes are cleared. Fig.?? is the wallpaper for stamp rally mode, and there are ten quiz icon at the bottom of the image.

\section{VISITING NAVIGATION}

In exhibition facilities with a large or complex exhibition fields, it is often heard of that visitors cannot find the exhibit position or visitors can not complete their planed visiting for they cannot find a correct path for all exhibit that they want to visit. Therefore, navigation service can solve the above claims and make the guests enjoy the visiting time greatly. In the research, we present an approach to provide exhibition navigation function. We provide the 


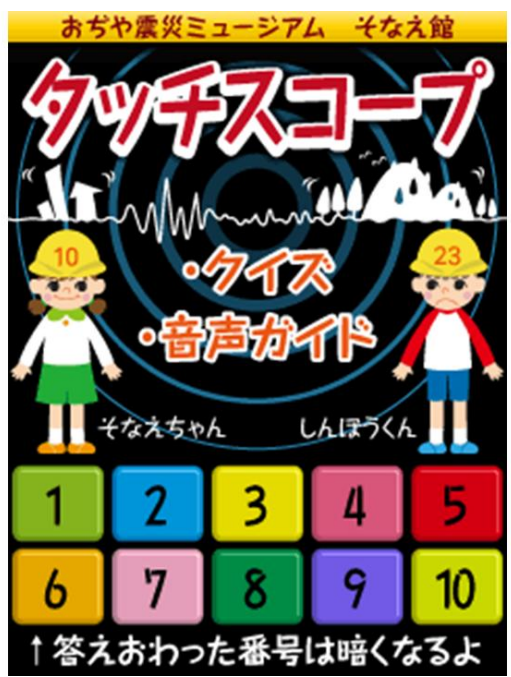

Fig. 9. Wallpaper for stamp rally mode

guidance by the ID signal from NFC tag, therefore, we locate the position by the NFC tag. There are three type NFC tag, RFID tag, IR tag, and PAN tag. RFID is a passive tag based on ISO15693 standard. IR tag is an active tag using IrDA physical layer. PAN tag is also an active tag using $2.4 \mathrm{GHz}$ band radio. table 3 hows the specification of each tag.

Table 3. The Specification of NFC Tag

\begin{tabular}{|l|l|l|l|}
\hline Specification Item & RFID tag & IR tag & PAN tag \\
\hline reading distance & $\leq 10 \mathrm{~cm}$ & $\leq 5 \mathrm{~m}$ & $\leq 5 \sim 15 \mathrm{~m}$ \\
\hline Media & Radio & Infrared & Radio \\
& $13.56 \mathrm{Mhz}$ & $780 \mathrm{~nm}$ & $2.4 \mathrm{GHz}$ \\
\hline Action Type & Passive & Active & Active \\
\hline Power Type & None & Wall Power & Battery \\
\hline
\end{tabular}

RFID can give highest accuracy, but it needs the visitor to touch the tag. Other two active tags can send out the ID signal periodically, therefore visitors will get the ID signal when they enter the valid area of the tag. To realize navigation, we need to create a data structure to save the relation between the ID and the position in the exhibition area. To describe the information about a spot, we define a vector $d$ in the following format.

$$
\begin{aligned}
d & =\langle P, I, L, M, R, S\rangle \\
P & =\left\langle\text { ID }_{\text {tag }}, \text { Map }_{\text {index }_{\text {f }}}, x, y\right\rangle \\
I & =\left\langle\text { Icon }_{\text {index }}, \text { Icon }_{\text {pos }}\right\rangle \\
L & =\left\langle\text { Label }_{\text {offset }}, \text { Label }_{\text {length }}, \text { Label }_{\text {mode }}\right\rangle \\
M & =\left\langle\text { Map }_{\text {orientation }}, \text { Map }_{\text {shape }}\right\rangle
\end{aligned}
$$

Here $P$ contains the information about the tag in a spot. In $P$, $I D_{\text {tag }}$ is the ID of the tag. $M a p_{\text {index }}$ is the index to the map. $x$ and $y$ are the coordinates of the spot in the map. $I$ contains information about the icon for the spot. In $I, I c o n_{\text {index }}$ and $I c o n_{\text {pos }}$ refer to the icon index and icon showing position. If $I \operatorname{con}_{\text {index }}=0$, it means that there is no icon ,but only a color square mark for the showing the spot on the map. $L$ contains the information about the label for the spot. In $L, L a b l e_{o f f s e t}$ and Lable length $_{\text {is }}$ used for get the label text from label file, which save the label in UNICODE. Therefore, the multi-language label can be shown in the map. Label $_{\text {mode }}$ will give setting information about showing the label. Finally, $M$ is the information about showing the map, Map orientation gives the rotation angle, and $M a p_{\text {shape }}$ gives the shape of the map, there are only two value for the shape, portrait or landscape. $P$ is the rating value for the exhibition. $S$ is the status for visiting. If the spot has been visited, $S=$ true, else $S=$ false. By modifying status in the table, we can track the visiting history of the visitor. Therefore, the set $D=\left\{d_{1}, d_{2}, \cdots, d_{N}\right\}$ is used to express all the exhibition spot. With the set, we can give the current exhibition status correctly.

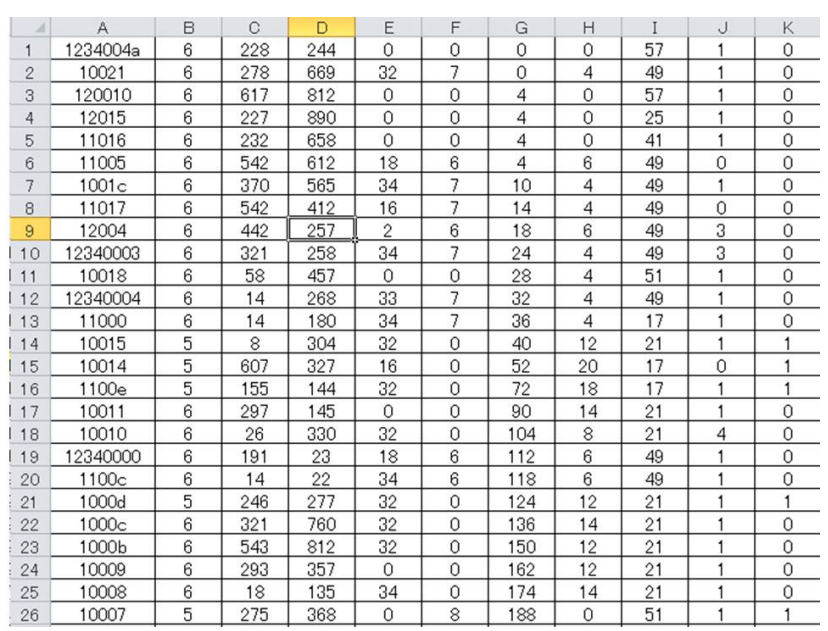

Fig. 10. A sample of the tag CSV file

The information about all the spot where a tag exists, will be saved in a CSV file as shown in Fig 10 it is a hard work to create the table file manually. To make it easier to edit the map, we developed map maker software to register the tag or the exhibit information, such as tag ID, position coordinates, and labels. Here we use a pure base image of the exhibition area. If the exhibition area is too large or there are several exhibition floors, we can split the map into several smaller ones. The portable terminal will switch the map automatically based on the table about spot information. Fig 11 and Fig 12 show the main GUI of the map maker in edit mode and preview mode respectively.

With the map date, the portable device can show the map based on the tag ID information and visiting history information. The map can be shown in two scale zoom. With button operation, the map can be shifted also. The icon of visited exhibit will be shown with a different one, therefore visitors can know what they have visited. For there is a 6D MEMS sensor on board, the screen will show the orientation of the user. This function can help the user to find the correct path for next exhibit that they will visit. There are two typical applications for location service. One is only showing the visitors where they are. Another service is visiting path navigation. The terminal will show the visitor the next possible exhibition spot that the visitor may be interested. 


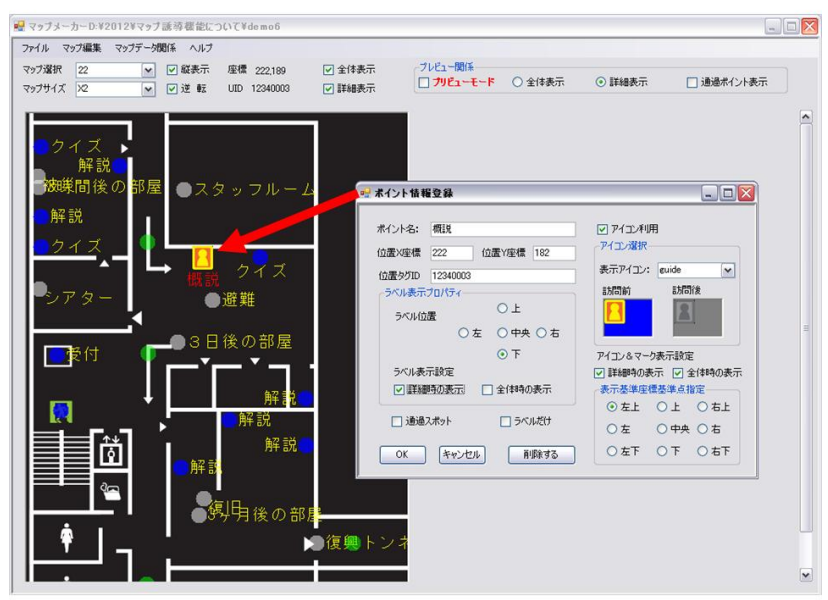

Fig. 11. GUI of the map maker in edit mode

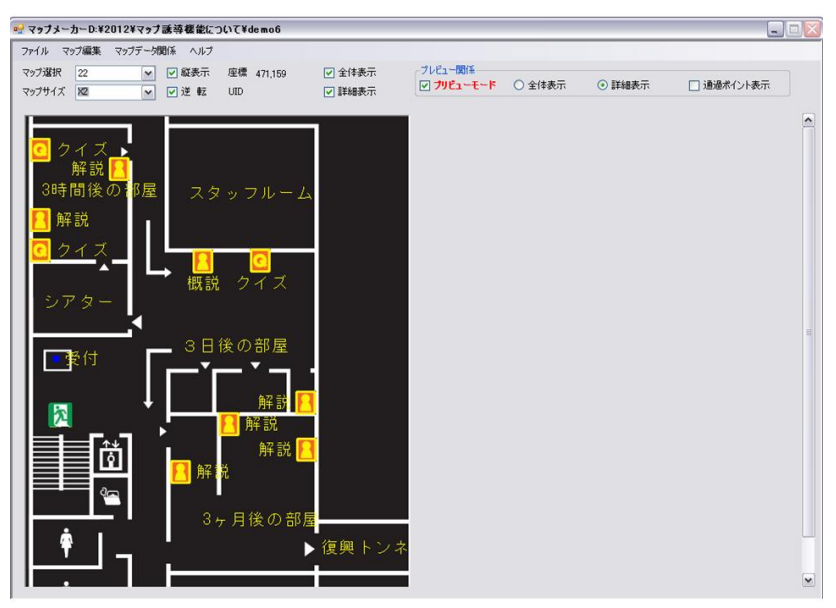

Fig. 12. GUI of the map maker in preview mode

\subsection{A Navigation based on Location and Popularity}

In big exhibition area, visitor would want to appreciate the popular exhibition in a limited time. As this case, exhibition navigation based on the location of the visitor is important. Here we give a navigation approach based on the position of the visitor and the popularity. To realize it, an evaluation function $J$ is defined as following

$$
\min _{\sigma} J=\sum_{i=1}^{n} \frac{\left(x_{\sigma(i-1)}-x_{\sigma(i)}\right)^{2}+\left(y_{\sigma(i-1)}-y_{\sigma(i)}\right)^{2}}{R_{\sigma(i)}}
$$

Here, $\sigma(i) \in\{1,2, \cdots, n\}$ is the sequence of visiting in order for $i=1,2,3, \cdots, n .1$ is for current position where the visitor is standing. $R_{\sigma(i)}$ refers to the popularity of the exhibition. This is a typical traveling salesman problem (TSP), and there are many algorithms for the solution. The candidate spots mainly selected from the neighborhood spots around the current position and they have not been visited yet. By using $R_{\sigma}$, a spot with a high popularity will be selected.

\section{EVALUATION}

The museum guidance system has been adopted by an earthquake museum in Japan. In the guidance system, up to 50 portable terminals are used to provide guidance service. In the exhibition area, there are about 100 exhibition spots. For each spot, an IC tag is set to send out ID information about the spot. When the visitor close to the spot, the guidance device will automatically start a guidance based on the ID. The terminal provides not only a multimedia guidance on the exhibition, but also guidance navigation by showing the map of the exhibition area. With the map, the visitors can confirm the position where they are standing, the visiting status about the exhibition spot and stamp rally spot. By collecting the visiting records, the exhibition popularity can be obtain that will be used in guidance navigation. Also, the visitors especially the youths are very interested with the new stamp rally service. Fig 13 is the scenes in the museum. A questionnaire was carried out to investigate the usage of the system. By the questionnaire, more than $85 \%$ visitors satisfied with the guidance by the system. It was confirmed that the system contributes to the service usefulness and customer satisfaction.

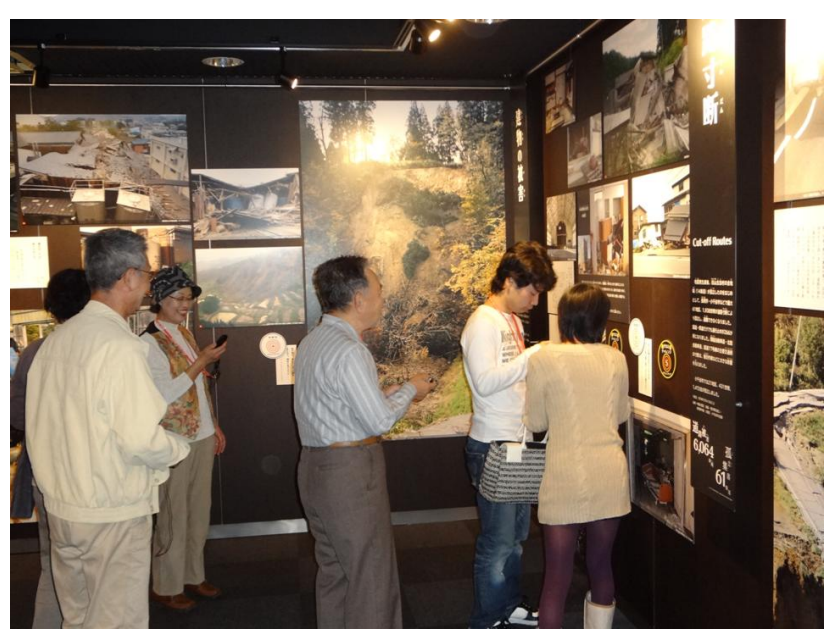

Fig. 13. Visitors are watching the guidance in the museum

\section{CONCLUSIONS}

This paper describes a new interactive museum guide system that provides both interactive guidance on the exhibition, and visiting navigation with an easy to use interface. With the information from NFC tags, the portable terminal of the system automatically starts a multimedia guidance with an interactive GUI, also displays a zoomed map view to show the visit's current position and visiting history status. For exhibition facilities, four sub support systems are also provided. The contents editor application makes the contents editing easier with only simple mouse operation. The map maker software provides an easy use environment to edit the spot data for navigation. The integration management station provides an efficient environment to update the portable terminals, and monitor the status of the device. The stamp rally control system make the 
facilities staff provide stamp rally service very easily. The new system had been already commoditized and adopted in several museums in Japan and a good performance has been confirmed. Future work will include the wearable device development by using the NFC and MEMS ability. With the localization technique and AI, we expect to develope a new wearable guidance system that can provide an automatic guidance service based on the location and status of the visitors.

\section{REFERENCES}

[1] Kusunoki, F.; Sugimoto, M.; Hashizume, H. Toward an interactive museum guide system with sensing and wireless network technologies, Wireless and Mobile Technologies in Education, 2002. Proceedings. IEEE International Workshop on Volume , Issue , 2002 Page(s): 99 - 102

[2] Li-Der Chou; Chia-Hsieh Wu; Shih-Pang Ho; ChenChow Lee; Jui-Ming Chen, Requirement analysis and implementation of palm-based multimedia museum guide systems , Advanced Information Networking and Applications, 2004. AINA 2004. 18th International Conference on Volume 1, Issue , 2004 Page(s): 352 - 357 Vol.1

[3] Bartneck, C., Masuoka, A., Takahashi, T., Fukaya, T. An Electronic Museum Guide in Real Use, Psychology of Aesthetics, Creativity, and the Arts, 1(2), 114-120, 2007

[4] Dawei Cai, Ryuuta Kawashima, Tadaaki Takehana and Haruki Takahashi An Infrared Digital Contents Broadcasting Service for Mobiles, WSEAS Trans. on Communication, Vol.2(4), 73-78, 2005

[5] Dawei Cai, Yuji Saito and Yoshihiko Abe, An Information Broadcasting System with Infrared data Communication Protocol, WSEAS Trans. on Communication, Vol.2(3), 228234, 2003

[6] Carmine Ciavarella, Fabio Paterno, Design Criteriafor Location-aware, Indoor, PDA applications, Proceedings of Fifth International Symposium onHuman Computer Interaction with Mobile Devicesand Services, Udine, Italy, 2003

[7] Yo-Ping Huang, Wei-Po Chuang, Improving theMuseumfs Service by Data Mining and Location-aware Approach,2004 IEEE IntemationalConference on Systems and Man and Cybemetics, 2004

[8] Tsung-Yu Liu, Tan-Hsu Tan, Yu-Ling Chu, The Ubiquitous Museum Learning Environment: Concept,Design, Implementation, and a Case Study, Proceedings of the Sixth International Conference onAdvanced Learning Technologies (ICALT'06), 2006 